\title{
The Origins of the French Revolution
}

Jean-Pierre Gross

\section{OpenEdition}

\section{Journals}

Édition électronique

URL : https://journals.openedition.org/ahrf/7983

DOI : 10.4000/ahrf.7983

ISSN : 1952-403X

Éditeur :

Armand Colin, Société des études robespierristes

Édition imprimée

Date de publication : 1 décembre 2006

Pagination : 180-185

ISSN : 0003-4436

Référence électronique

Jean-Pierre Gross, «The Origins of the French Revolution », Annales historiques de la Révolution

française [En ligne], 346 | Octobre/Décembre 2006, mis en ligne le 10 juillet 2008, consulté le 23 avril 2022. URL : http://journals.openedition.org/ahrf/7983 ; DOI : https://doi.org/10.4000/ahrf.7983

Ce document a été généré automatiquement le 23 avril 2022.

Tous droits réservés 


\title{
The Origins of the French Revolution
}

\author{
Jean-Pierre Gross
}

\section{RÉFÉRENCE}

Peter R. Campbell (ed.), The Origins of the French Revolution, Basingstoke et New York, Palgrave Macmillan, 2006, 371 p., ISBN 0-333-94971-4, $18.99 £$.

Dimension religieuse captée sur la longue durée, genèse intellectuelle et culturelle, grippages et scléroses de la monarchie, arcanes décisionnels et impasses gestionnaires, déboires financiers et fiscaux, complexités géographiques du monde rural, crise de "l'État baroque» appréhendée dans toute son amplitude et reflétée dans l'iconographie contemporaine: tels sont les aspects saillants de cette remise en question des origines de la Révolution française. Ouvrage collectif présenté par Peter Robert Campbell de l'Université de Sussex, à la tête d'une équipe d'éminents chercheurs anglais et américains, et qui rassemble une dizaine d'essais, signés Dale Van Kley, John Markoff, Joël Félix, John Hardman, Kenneth Margerison, Michael Fitzsimmons, William Scott, Marisa Linton et Mark Ledbury.

Dans une contribution particulièrement stimulante, Dale Van Kley nous livre une vaste fresque religieuse qui repose sur cinq repères chronologiques : 1593, 1693, 1713, 1762 et 1791. Entre le «Paris vaut bien une messe » (apocryphe) de Henri IV et la Constitution civile du clergé comme bastion d'un gallicanisme radical, Van Kley reconnait dans ces marqueurs religieux la marche d'une monarchie irrémédiablement catholique, mais qui se voit progressivement désacralisée. Si le calvinisme s'avère dès ses origines incompatible avec la monarchie française, la lutte contre les places-fortes des réformés menée par Richelieu, suivie de la persécution des huguenots par Louis XIV, provoque la formation d'une diaspora protestante autour d'une France en guerre avec les principaux États européens. Or, l'absolutisme louisquatorzien a pour effet de susciter l'émergence d'un gallicanisme «national » aux dépens du gallicanisme royal : c'est-à- 
dire d'un parlementarisme porte-parole de l'hétérodoxie janséniste et défenseur des fameuses libertés gallicanes. Dans la mesure où le long conflit politico-religieux qui résulte de la condamnation pontificale du jansénisme provoque l'érosion de l'absolutisme bourbon et le démantèlement de son caractère sacral, le siècle des Lumières s'avère être en premier lieu le siècle d'Unigenitus. D'autant plus que les émotions populaires qui accompagnent ce conflit se polarisent autour de quartiers parisiens tels que le faubourg Saint-Marcel, foyer des futurs sans-culottes. Et pendant que les parlementaires élaborent un constitutionnalisme face au despotisme royal, le jansénisme, délaissant le corps épiscopal pour imprégner les rangs du bas-clergé, légitime une version radicalement gallicane de l'Église, qui place l'infaillibilité dans la masse des fidèles, prêtres et laïcs devenus témoins d'une nouvelle vérité constitutionnelle. Le conflit engendré par Unigenitus oppose l'autorité du trône à celle de l'État et finit par désacraliser la monarchie. À ce conflit vient s'ajouter la lutte acharnée contre la Compagnie de Jésus, puissance « étrangère » assujettie à la papauté et dont la constitution ultramontaine, proche du despotisme de Louis XIV, est incompatible avec les principes du gallicanisme. Le séquestre des biens des jésuites, qui précède leur expulsion, n'est pas sans préfiguer les réformes ecclésiastiques de l'Assemblée nationale en 1791, imbues de nationalisme " gallican ». La guerre religieuse entre jansénistes et jésuites attise les feux des Lumières, comme l'attestent les contes de Voltaire, et le caractère anticlérical, voire antichrétien, du mouvement philosophique préfigure le radicalisme déchristianisateur de la Révolution. De même, après l'épuration des parlements entrepris par Maupeou, les préjugés religieux se politisent encore plus, le parti janséniste se mutant en parti patriote, le parti dévot s'érigeant en défenseur de l'autorité indivise. Mais au dire de Dale Van Kley, qui rejoint ici l'approche de Monique Cottret, l'opinion publique française eût joyeusement accueilli, à n'importe quel moment entre 1715 et 1789 , la transformation de la monarchie de droit divin en monarchie par la volonté du peuple, la Nation souveraine pouvant prendre la place d'un roi fragilisé à la tête de l'église gallicane, en se dispensant de l'autorité spirituelle de Rome. Et l'anathème du pape jeté sur la Constitution civile du clergé s'explique par le caractère souverain et démocratique de l'autorité nationale, alors qu'aucune censure pontificale ne vint frapper la législation ecclésiastique de l'empereur Joseph II, pourtant presque aussi draconienne, mais qui relevait de l'autorité impériale. La fuite de Louis XVI arrêtée à Varennes en 1791 trahit, ironiquement, un choix en faveur d'un certain type de catholicisme et constitue un lointain rappel du choix de Henri IV en 1593, sauf qu'à présent le roi n'obéit plus à la volonté de son peuple : Henri, quant à lui, avait refusé de convoquer les États généraux, mais respectait la volonté de la grande majorité de ses sujets catholiques.

3 Si Dale Van Kley met en lumière les aléas de l'absolutisme de droit divin, les auteurs de plusieurs chapitres viennent braquer le projecteur sur les faiblesses politiques de l'État baroque : tels John Hardman, Peter Robert Campbell, Kenneth Margerison et Michael Fitzsimmons. Et ce par des exemples ponctuels, cueillis dans la courte durée, mais très parlants. John Hardman, auteur d'une remarquable biographie de Louis XVI, et qui nous a livré la Correspondance de Louis XVI et de Vergennes, examine les structures du processus décisionnel de la monarchie et se demande comment le roi, toujours indécis, en vint infailliblement à prendre les mauvaises décisions ou n'en prit aucune quand elles s'imposaient. Loin d'entériner la légende de "l'État administratif moderne ", Hardman dissèque les arcanes gouvernementaux de la cour pour en révéler les faiblesses et scléroses : tel le système de gouvernement par comités interposés, institué 
par Maurepas entre 1774 et 1785 pour court-circuiter le conseil d'État, et neutraliser l'influence des factions, groupées autour de Miromesnil, Castries, Vergennes, Calonne, la côterie Polignac ou "société intime de la reine». En quoi les procédures de consultation ont-elles pu empêcher la monarchie française de sombrer dans un despotisme de type ottoman? Castries fit valoir que le roi préférait l'unanimité à un vrai débat, puisqu'elle lui permettait de mieux dormir la nuit. Certes, le réveil serait rude! Sans doute, concède Hardman, certaines décisions étaient nécessaires, d'autres ne l'étaient pas. Ainsi, trois décisions du règne de Louis XVI - le rappel du parlement en 1774, l'alliance signée avec les Américains en 1778 et la convocation de l'assemblée des notables en 1786 - n'avaient pas à être prises et entraînèrent des conséquences à haut risque. Voici donc un roi qui répugnait à prendre des décisions, mais qui crut devoir en prendre et provoqua par là les conséquences mêmes qu'elles étaient censées éviter. Le remède proposé par Calonne à la crise financière et soumis aux notables révélait un déficit qui exposait le royaume à la faillite, mais sans véritable stratégie qui eût permis de sortir de la crise. Isolés, le roi et son ministre, serviteur exemplaire de la monarchie absolue, provoquaient une levée de boucliers de l'ordre établi. Le roi léthargique du mythe historique s'affirmait volontariste et despotique malgré lui, et mériterait plutôt le qualificatif de « Louis le téméraire »!

4 Peter Robert Campbell souligne à son tour l'intérêt d'une telle démarche : aborder la problématique gouvernementale par le biais des relations entre couronne et parlement lui permet de mieux comprendre comment les conflits furent générés et résolus, et quel fut le rôle respectif dans ce processus de ministres et magistrats, compte tenu des filières administratives, des problèmes de juridiction, des faveurs royales, du clientélisme, des intrigues des factions, et de la rhétorique. Le parlement de Paris subit entre 1771 et 1788 des épreuves qui auraient pu en faire le champion du radicalisme, mais le corporatisme l'emporta. Comme Bailey Stone l'a démontré, les magistrats étaient foncièrement conservateurs, attachés au principe de la division de la société en trois ordres et au bien-fondé du privilège. Sur le plan politique, ils affichèrent un " royalisme ambivalent ", dévoués qu'ils étaient à la monarchie, tout en l'empêchant de fonctionner. Les parlements assumaient le rôle de pouvoirs intermédiaires, nécessaires à l'équilibre de la société et devant servir de rempart au despotisme. C'est ainsi que le parlement de Paris fut amené à signer son arrêt de mort le 25 septembre 1788, en décrétant que les États généraux se réuniraient selon les formes de 1614, et en se rangeant ainsi du côté des deux ordres privilégiés. Du jour au lendemain, sa popularité et son prestige, qui étaient immenses aux yeux de l'opinion publique, s'évanouirent : le Tiers état vit dans cette décision la volonté de défendre le statu quo et les privilèges de la noblesse, l'abandon de la lutte contre l'absolutisme, un renoncement à la mission de régénérer la France. La crise de 1787-1788 ressemble aux crises antérieures entre couronne et parlement, où l'intrigue et le bluff jouaient un rôle prépondérant, mais cette fois-ci, souligne Campbell, les ministres ne réussirent pas à obtenir le rééchelonnement de la dette et furent obligés de compromettre pour toujours l'autorité royale, tandis que l'aveuglement des parlementaires les empêcha de saisir les enjeux historiques du moment: de part et d'autre, on enregistre un échec massif de gestion politique, lourd de conséquences.

5 Avis partagé par Kenneth Margerison, qui examine le débat pamphlétaire sur l'organisation des États généraux, alimenté par le Qu'est-ce que le Tiers état ? de l'abbé Sieyès, et qui déboucha sur l'union des trois ordres et la création d'une Assemblée nationale représentative de la Nation entière. Margerison met en lumière l'action de 
Duport et de la société des Trente, de Mounier, Target, et Barnave, qui rassemblèrent les partisans de l'union. La nuit du 4 Août vit le triomphe de ce parti, regroupé autour des représentants de la noblesse libérale, et qui orchestra ensuite la rédaction de la Déclaration des droits de l'homme et du citoyen, aux dépens de Sieyès, dont l'influence diminua sensiblement. L'analyse de Margerison remet en question à la fois l'interprétation de Georges Lefebvre, qui fit le portrait d'une Assemblée dominée par le Tiers, et celle de François Furet et de Keith Baker, qui ont chacun souligné le rôle prépondérant de Sieyès dans l'élaboration du concept de souveraineté et de citoyenneté. Sur cette même lancée, Michael Fitzsimmons étudie avec minutie le déroulement des événements depuis l'ouverture des États généraux le 5 mai 1789 jusqu'à l'appropriation de la souveraineté nationale par l'Assemblée au cours du mois de juin. Il met en évidence la perte de l'autorité royale en raison du vide même qui régna à la suite de l'absence d'initiative de la part du roi. Au grand désarroi des députés, celui-ci n'esquissa aucun programme dans son discours d'ouverture, et lorsqu'il le fit le 23 juin, les risques d'une guerre civile eclipsèrent son projet. On assiste non à une volonté délibérée de la part des élus de défier la volonté royale, mais à une dérive progressive où la couronne perd le contrôle des événements. Fitzsimmons souligne que les premières semaines de l'Assemblée furent marquées par des velléités et sa seule réalisation concrète, le rapport du Comité de constitution du 27 juillet, fut d'une timidité désolante, refusant de condamner les lettres de cachet et acceptant la dilution ou le partage de la souveraineté. Seules la prise de la Bastille et les révoltes paysannes propulsèrent sur le devant de la scène la réalité d'une révolution populaire, consacrée enfin par l'abolition des privilèges dans la nuit du 4 Août. La genèse de l'idéal révolutionnaire fut lente à se manifester.

Abordant le sujet des crises financières d'Ancien Régime, Joël Félix fait remarquer que le débat autour des origines financières de la Révolution française a peu changé depuis 1789. Il en retrace l'évolution à partir de 1715 , tableaux à l'appui (financement de la guerre de la Succession d'Autriche, dépenses en temps de guerre, recettes annuelles moyennes et extraordinaires entre 1716 et 1788, emprunts de1740 à 1783), et partage l'avis de François Crouzet, selon lequel l'Ancien Régime s'est écroulé non pas en raison de la faiblesse de son économie, mais à cause d'une mauvaise gestion de ses finances. Les ministres de Louis XV et de Louis XVI n'ont pas su émuler l'exemple britannique donné par Walpole et Pelham, et créer un système viable de reconversion de la dette, avant de plonger le pays dans les guerres de Sept Ans et de l'Indépendance américaine. Or, J. Félix s'intéresse surtout aux retombées sociales et économiques de la politique fiscale des Bourbon. Il ne croit pas au mythe de la "taille arbitraire " destinée à opprimer le peuple, mythe engendré par Vauban et Boisguilbert et réitéré à satiété par une cohorte de philosophes au fil du siècle : au contraire, la crise de la monarchie ne résulte pas d'une politique fiscale injuste, mais des obstacles qu'elle a rencontrés chaque fois qu'elle a voulu introduire de nouveaux impôts plus équitables. Elle a tenté sans relâche d'abolir les privilèges fiscaux, se heurtant à chaque coup à la résistance farouche des privilégiés : capitation en 1695, dixième en 1710 (qui provoqua les foudres du duc de Saint-Simon), ensuite la longue série des vingtièmes, de telle sorte que les nobles, sinon le clergé, ont fini par se résigner à devenir contribuables : à partir de 1756, ils versaient au fisc en temps de paix, sous forme d'impôt direct, entre 10 et $12 \%$ de leur revenu net. Sans doute le foisonnement de taxes, directes et indirectes, créait une inertie qui entravait de tels efforts, et toute imposition nouvelle, telle la subvention territoriale, universelle et équitable, proposée par Calonne en 1786-1787, 
rencontrait un mur de refus. Néanmoins, les cahiers de doléances démontrent clairement qu'à quelques exceptions près (Normandie, Auvergne), la révolution fiscale était accomplie dans les cœurs avant même la réunion des États généraux. D'un commun avis, la gabelle était vouée à disparaitre. Quant aux réformes soumises par Calonne à l'assemblée des notables, elles portaient au grand jour l'ampleur de la crise financière, sans que l'on pût juger du montant exact du déficit. Ni Calonne, ni Necker, ni le roi, ni le parlement ne crièrent victoire, et l'on assista à la désintégration des doctrines dans la plus grande confusion. Le gouvernement ne pouvait continuer à emprunter sans augmenter les taxes, et en même temps conserver un système fiscal condamné par tous, gouvernement et opposition. Restait à savoir comment on s'y prendrait pour créer l'égalité devant l'impôt et un fisc comptable envers la Nation.

7 Saluons l'excellent résumé des revendications paysannes que nous offre John Markoff, grande autorité en la matière. Les cahiers de doléances constituent en effet une source d'une richesse exceptionnelle pour ce qui concerne la volonté exprimée des villageois de France. Markoff compare les données qu'il a dépouillées avec les nombreux incidents survenus pendant deux périodes circonscrites, 1690 à 1720 et 1760 à 1789 : les émeutes frumentaires l'emportent à présent sur les révoltes antifiscales. Si les paysans disent se contenter d'une réforme de la taille et de la dîme, ils commencent à exiger l'abolition pure et simple des droits seigneuriaux, sans compensation, puis se préparent à attaquer les châteaux et à demander la destruction des titres féodaux. Mais Markoff nous met en garde contre toute généralisation: conscient de la complexité géographique du royaume, il fait sienne la remarque de Pierre Goubert au sujet des vingt paysanneries de France. Il insiste sur la prise de conscience rapide des enjeux chez les villageois au printemps de 1789 , quand les cahiers se préparent, et sur la célérité avec laquelle les campagnes passent à l'action en juillet et août 1789. Il nous rappelle par là l'important corpus de travaux sur le monde rural et la révolution paysanne que nous ont laissé nos maîtres Marc Bloch, Georges Lefebvre, Albert Soboul, Anatoli Ado, et leurs disciples. Parmi les chercheurs actuels, il rend hommage également à Peter Jones, Steven Kaplan, Cynthia Bouton et Jean-Pierre Jessenne. Réciproquement, nous sommes tous redevables envers John Markoff pour la magistrale analyse du contenu des cahiers de doléances qu'il a fait paraître en 1998 avec son collègue Gilbert Shapiro, au terme de plus de quatre décennies de recherches exhaustives.

8 Nous évoquerons pour terminer les chapitres consacrés à l'histoire intellectuelle et culturelle, de William Scott, Marisa Linton et Mark Ledbury. Pour le lecteur non encore rassasié des querelles d'école qui ont accompagné et suivi le bicentenaire, des références à la "sphère publique » selon Jürgen Habermas, ou des différents registres $\mathrm{du}$ « discours politique » selon Keith Baker, nous saurons gré à ces trois auteurs de fournir une synthèse claire d'un sujet souvent hermétique. À ce débat d'idées, Mark Ledbury apporte une agréable contribution iconographique et théâtrale, où il approfondit la "rhétorique visuelle » et décrit la "sphère culturelle française ", qui enjambe Ancien Régime et Révolution. Il passe ainsi du drame bourgeois et de l'Accordée de village (1761) de Jean-Baptiste Greuze au Serment des Horaces (1785), puis au Triomphe du peuple français (1793) de Jacques-Louis David, pour démontrer comment la sphère du visuel échappe progressivement à l'emprise royale, pour devenir espace où le spectateur découvre de nouvelles valeurs, telles que vertu et patriotisme. Mais pourquoi faut-il que le portrait de Marie-Antoinette et ses enfants (1787) par Elisabeth Vigée-Lebrun serve de prétexte à l'auteur pour nous entretenir du «revers de la 
médaille", soit les représentations pornographiques de la reine dans les images clandestines circulant à la veille de la Révolution ? N'en déplaise à Robert Darnton et à Lynn Hunt, ce portrait de la famille royale, qui date de 1787, réussit magnifiquement à promouvoir une vision aimable de la monarchie, hors du temps et à l'abri du discrédit à venir. Mais qui traite des origines de la Révolution française aborde un sujet inépuisable, où tout prétexte est bon pour ouvrir de nouvelles perspectives et provoquer de nouvelles controverses. Ce volume à facettes multiples enrichit un débat qui est encore loin d'être clos. 